\title{
Insulin-like growth factor I and risk of epithelial invasive ovarian cancer by tumour characteristics: results from the EPIC cohort
}

\author{
J Ose $^{1}$, R T Fortner ${ }^{1}$, H Schock ${ }^{1}$, P H Peeters ${ }^{2}$, N C Onland-Moret ${ }^{2}$, H B Bueno-de-Mesquita $3,4,5$, \\ E Weiderpass ${ }^{6,7,8,9}$, I T Gram $^{10}$, K Overvad $^{11}$, A Tjonneland ${ }^{12}$, L Dossus ${ }^{13,14,15}$, A Fournier ${ }^{13,14,15}$, \\ L Baglietto ${ }^{16,17}$, A Trichopoulou ${ }^{18,19}$, V Benetou ${ }^{19,20}$, D Trichopoulos ${ }^{18,19,21}$, H Boeing ${ }^{22}$, G Masala ${ }^{23}$,
} V Krogh ${ }^{24}$, A Matiello ${ }^{25}$, R Tumino ${ }^{26}$, M Popovic $^{27}$, M Obón-Santacana ${ }^{28}$, N Larrañaga ${ }^{29,30}$, E Ardanaz ${ }^{30,31}$, M-J Sánchez ${ }^{30,32}$, V Menéndez ${ }^{33}, \mathrm{M}-\mathrm{D}$ Chirlaque ${ }^{30,34}$, R C Travis ${ }^{35}$, K-T Khaw ${ }^{36}$, J Brändstedt ${ }^{37}$, A Idahl ${ }^{38}$, E Lundin ${ }^{39}$, S Rinaldi ${ }^{40}$, E Kuhn ${ }^{40}$, I Romieu ${ }^{40}$, M J Gunter ${ }^{41}$, M A Merritt ${ }^{41}$, E Riboli ${ }^{41}$ and R Kaaks ${ }^{\star}, 1$

${ }^{1}$ Division of Cancer Epidemiology, German Cancer Research Center, 69120 Heidelberg, Germany; ${ }^{2}$ Department of Epidemiology, Julius Center for Health Sciences and Primary Care, University Medical Center Utrecht, 3584 Utrecht, The Netherlands; ${ }^{3}$ National Institute for Public Health and the Environment (RIVM), 3720 Bilthoven, The Netherlands; ${ }^{4}$ Department of Gastroenterology and Hepatology, University Medical Centre, 3542 Utrecht, The Netherlands; ${ }^{5}$ Department of Epidemiology and Statistics, the School of Public Health, Imperial College London, SW72AZ London, UK; ${ }^{6}$ Department of Community Medicine, Faculty of Health Sciences, University of Tromsø, The Arctic University of Norway, 90109 Tromsø, Norway; ${ }^{7}$ Cancer Registry of Norway, 0304 Oslo, Norway; ${ }^{8}$ Department of Medical Epidemiology and Biostatistics, Karolinska Institutet, 17177 Stockholm, Sweden; ${ }^{9}$ Department of Genetic Epidemiology, Folkhälsan Research Center, 00014 Helsinki, Finland; ${ }^{10}$ Department of Community Medicine, Faculty of Health Sciences, UiT The Arctic University of Norway, 9037 Tromsø, Norway; ${ }^{11}$ Department of Public Health, Section for Epidemiology, Aarhus University, 8000 Aarhus, Denmark; ${ }^{12}$ Institute of Cancer Epidemiology, Danish Cancer Society Research Center, 2100 Copenhagen, Denmark; ${ }^{13}$ Inserm, Centre for research in Epidemiology and Population Health (CESP), U1018, Nutrition, Hormones and Women's Health team, F-94805 Villejuif, France; ${ }^{14}$ Univ Paris Sud, UMRS 1018, F-94805 Villejuif, France; ${ }^{15}$ IGR, F-94805 Villejuif, France; ${ }^{16}$ Cancer Epidemiology Centre, Cancer Council of Victoria, Melbourne, 3004 Victoria, Australia; ${ }^{17}$ Centre for Epidemiology and Biostatistics, School of Population and Global Health, University of Melbourne, Melbourne, 3004 Victoria, Australia; ${ }^{18}$ Bureau of Epidemiologic Research, Academy of Athens, 23 Alexandroupoleos Street, Athens GR-115 27, Greece; ${ }^{19}$ Hellenic Health Foundation, 13 Kaisareias Street, Athens GR-115 27, Greece; ${ }^{20}$ Department of Hygiene, Epidemiology and Medical Statistics, University of Athens Medical School, 75M Asias Street, Goudi, Athens GR-115 27, Greece; ${ }^{21}$ Department of Epidemiology, Harvard School of Public Health, 677 Huntington Avenue, Boston, MA 02115, USA; ${ }^{22}$ Department of Epidemiology, German Institute of Human Nutrition (DIfE) 14558 Potsdam-Rehbrücke, Nuthetal, Germany; ${ }^{23}$ Molecular and Nutritional Epidemiology Unit, Cancer Research and Prevention Institute-ISPO, 50139 Florence, Italy; ${ }^{24}$ Epidemiology and Prevention Unit, Fondazione IRCCS Instituto Nazionale dei Tumori, Via Veneziani 1, 20133 Milano, Italy; ${ }^{25}$ Department of Clinical and Experimental Medicine, Federico II University, 80131 Naples, Italy; ${ }^{26}$ Cancer Registry and Histopathology Unit, 'Civic - M.P. Arezzo' Hospita, ASP 97100 Ragusa, Italy; ${ }^{27}$ Unit of Cancer Epidemiology, AO Citta' della Salute e della Scienza, Department of Medical Sciences, University of Turin and Center for Cancer Prevention (CPO-Piemonte), 10126 Turin, Italy; ${ }^{28}$ Unit of Nutrition, Environment and Cancer, Cancer Epidemiology Research Program, Catalan Institute of Oncology (ICO-IDIBELL), 08908 Barcelona, Spain; ${ }^{29}$ Public Health Division of Gipuzkoa-BIODonostia Research Institute, Basque Regional Health Department, 20013 San Sebastian, Spain; ${ }^{30}$ Consortium for Biomedical Research in Epidemiology and Public Health (CIBER), 28029 Madrid, Spain; ${ }^{31}$ Navarre Public Health Institute, 31006 Pamplona, Spain; ${ }^{32}$ Andalusian School of Public Health, 18011 Granada, Spain; ${ }^{33}$ Public Health Directorate, 33006 Asturias, Spain;

*Correspondence: Dr R Kaaks; E-mail: r.kaaks@dkfz-heidelberg.de

Received 28 May 2014; revised 24 September 2014; accepted 8 October 2014; published online 4 November 2014 (c) 2015 Cancer Research UK. All rights reserved 0007-0920/15 
${ }^{34}$ Department of Epidemiology, Murcia Regional Health Authority, 30008 Murcia, Spain; ${ }^{35}$ Cancer Epidemiology Unit, University of Oxford, OX30NR Oxford, UK; ${ }^{36}$ Department of Public Health and Primary Care, University of Cambridge, CB22QQ Cambridge, UK; ${ }^{37}$ Medical Department of Surgery, Malmö University Hospital, 20502 Malmö, Sweden; ${ }^{38}$ Department of Clinical Sciences, Obstetrics and Gynecology and Department of Public Health and Clinical Medicine, Nutritional Research Umeå University, 90185 Umeå, Sweden; ${ }^{39}$ Department of Medical Biosciences, Pathology Umeå University, 90185 Umeå, Sweden; ${ }^{40}$ International Agency for Research on Cancer, 69372 Lyon, France; ${ }^{41}$ Department of Epidemiology and Biostatistics, School of Public Health, Imperial College, SW72AZ London, UK

Background: Prospective studies on insulin-like growth factor I (IGF-I) and epithelial ovarian cancer (EOC) risk are inconclusive. Data suggest risk associations vary by tumour characteristics.

Methods: We conducted a nested case-control study in the European Prospective Investigation into Cancer and Nutrition (EPIC) to evaluate IGF-I concentrations and EOC risk by tumour characteristics ( $n=565$ cases). Multivariable conditional logistic regression models were used to estimate associations.

Results: We observed no association between IGF-I and EOC overall or by tumour characteristics.

Conclusions: In the largest prospective study to date was no association between IGF-I and EOC risk. Pre-diagnostic serum IGF-I concentrations may not influence EOC risk.

Insulin-like growth factor I (IGF-I)-related signalling pathways are implicated in the aetiology of epithelial cancer at various sites (e.g., prostate, colon and breast cancer), including ovarian cancer (reviewed in Bruchim and Werner, 2013; Singh et al, 2014). Insulin-like growth factor I drives cellular proliferation in several cell lines of epithelial neoplasms (reviewed in Pollak, 2008) and is additionally associated with invasion and angiogenesis in epithelial ovarian cancer cells (reviewed in Beauchamp et al, 2010). Recently, IGF-I was shown to be overexpressed in low-grade, but not highgrade, serous ovarian cancer cell lines, suggesting IGF-I may be differentially associated with risk across ovarian cancer subtypes (King et al, 2011). Further, low-grade ovarian cancer cells expressing IGF-I were more responsive to IGF-I stimulation and IGF-I receptor (IGF-IR) inhibition compared with high-grade serous ovarian cancer cells (King et al, 2011).

Prior prospective studies on the association between prediagnostic circulating IGF-I and epithelial invasive ovarian cancer (EOC) were inconclusive and evaluated EOC as a single disease entity, without addressing associations in EOC subgroups (i.e., histologic subtype, dualistic model of ovarian carcinogenesis) (Lukanova et al, 2002; Peeters et al, 2007; Tworoger et al, 2007). This is the largest prospective study to date ( $n=565$ cases; 1097 controls) investigating the role of IGF-I and EOC risk, and the first prospective investigation to assess IGF-I and EOC by tumour characteristics (histology, grade, stage and type I/II status).

\section{MATERIALS AND METHODS}

Study population. The European Prospective Investigation into Cancer and Nutrition (EPIC) is an ongoing multicentre prospective cohort study. Descriptions of study design, population and baseline data collection of the cohort (Riboli et al, 2002) and this nested casecontrol study (Ose et al, 2014) have been reported in detail. Briefly, 519978 participants (366 521 women) aged 25-70 years were enrolled from 1992 to 2000 in 10 European countries. Data on diet, reproductive factors, use of exogenous hormones (oral contraceptives (OC) and hormone replacement therapy (HRT)), disease history and anthropometric measures were collected at baseline. A total of 226673 women provided a baseline blood sample.

Women not using exogenous hormones at blood donation and with no history of cancer at recruitment (with the exception of non-melanoma skin cancer) were eligible for this study.
A total of 565 eligible cases with biological samples and incident epithelial invasive ovarian, fallopian tube or primary peritoneal cancer were 1:2 matched to 1097 controls. An incidence density sampling protocol was used. We included 201 cases and 372 matched controls from a prior analysis in EPIC (phase 1; Peeters et $a l, 2007)$ and additional 364 cases (725 matched controls) subsequently diagnosed during follow-up (phase 2).

Information on tumour characteristics (histologic subtype (serous, endometrioid, clear cell, mucinous and not otherwise specified (NOS)), grade (well, moderately or poorly/undifferentiated) and stage (local, regional and metastatic)) was available from pathology reports and from cancer registries. Tumours were classified on the basis of histology and the proposed dualistic pathway of ovarian carcinogenesis (type I/II; Kurman and Shih, 2011). Clear cell carcinomas $(n=28)$ were excluded from type I/II analyses as they show unique clinical behaviour (Penson et al, 2013). All participants gave written informed consent. The Ethical Review Board of the International Agency for Research on Cancer and the Institutional Review Board of each EPIC centre approved these analyses.

Laboratory assays. Pre-diagnostic concentrations of IGF-I $\left(\mathrm{nmoll} \mathrm{l}^{-1}\right)$ were analysed with enzyme-linked immunosorbent assays at IARC (phase 1 (Peeters et al, 2007): DSL, Webster, TX, USA) and at the Division of Cancer Epidemiology at the German Cancer Research Center (phase 2: Immunodiagnostics Systems, Frankfurt, Germany). Cases and matched controls were analysed within the same analytical batch by laboratory technicians blinded to case-control status and identity of quality control samples. Intra- and inter-batch coefficients of variation from replicate quality control (QC) samples were $2.50 \%$ and $12.20 \%$ (phase 1: triplicate QCs), and 9.42\% and 8.93\% (phase 2: duplicate QCs).

Statistical analyses. Insulin-like growth factor I values were $\log 2$ transformed and centred to a mean value of zero in each phase. Odds ratios (ORs) and 95\% confidence intervals (CIs) were calculated using conditional logistic regression. Insulin-like growth factor I was examined continuously on the $\log 2$ scale and in tertiles with phase-specific cut-offs based on the distribution in controls.

The final model included full-term pregnancy (never/ever), as other covariates (BMI, height, smoking, physical activity, diabetes, alcohol, age at menarche, age at first birth, number of births, age at menopause, OC use and HRT use) did not change the OR by $>10 \%$ (i.e., by a factor 1.10 or its reciprocal; Maldonado and Greenland, 1993). 
Heterogeneity in the associations between IGF-I and EOC by tumour characteristics was assessed using likelihood ratio tests comparing logistic regression models with and without corresponding interaction terms (Rothman et al, 2008).

Sensitivity analyses included stratification by menopausal status at blood donation and age at diagnosis ( $<55$ and $\geqslant 55$ years); exclusion of women providing a blood sample $<2$ years before diagnosis (to ensure any observed associations were not due to cancers influencing circulating concentrations of IGF-I, but not yet diagnosed) and women who had a prior hysterectomy.

All statistical tests were two-tailed and significant at the $P<0.05$ level. SAS 9.2 (SAS Institute Inc., Cary, NC, USA) was used for all statistical analysis.

\section{RESULTS}

Cases and controls were similar with respect to most characteristics, except for established reproductive risk factors (e.g., cases were less likely to be parous, $P<0.01$ or to use OCs, $P<0.01$; Table 1$)$. We observed no case-control differences in IGF-I concentrations overall or by study phase.

There was no association between EOC and IGF-I concentrations for doubling of hormone concentrations or comparing top to bottom tertiles in overall analyses (all histological subtypes combined $\left(\mathrm{OR}_{\log 2}=0.88 ; 95 \%\right.$ CI $\left.\left.0.71-1.08\right)\right)$. A similar pattern was observed in subgroup analyses by tumour characteristics (e.g., serous tumours: $\mathrm{OR}_{\log 2}=0.98 ; 95 \%$ CI $0.74-1.30$; Table 2). We did not observe heterogeneity between risk associations by tumour characteristics (e.g., $P_{\text {het }}$ for histological subtypes $=0.12$ ). Overall, risk estimates were similar when analyses were restricted to study phase 2 (data not shown).

Results were similar in sensitivity analyses by age at diagnosis $(<55 v s \geqslant 55)$ and menopausal status at blood donation (pre- $v s$ postmenopausal at blood donation). Excluding women with unilateral oophorectomy/hysterectomy $(n=116)$ or women diagnosed within 2 years after blood donation $(n=84)$ led to results comparable to overall results (data not shown).

\section{DISCUSSION}

This is the largest prospective study on the relationship between IGF-I and EOC to date and the first to assess risk associations by tumour characteristics. We observed no association between IGF-I and EOC overall. The same pattern was observed in analyses stratified by tumour characteristics, age at diagnosis or menopausal status at blood donation.

Three prospective studies (range: 132 cases (Lukanova et al, 2002) to 222 cases (Tworoger et al, 2007)) have evaluated this association previously. Two of these studies observed a positive association between IGF-I and EOC in women $<55$ at diagnosis (Lukanova et al, 2002; Peeters et al, 2007); however, sample size in these subgroups was limited ( $n \leqslant 66$ younger than 55 at diagnosis) and confidence intervals were wide (i.e., $\mathrm{OR}_{\mathrm{Q} 3-\mathrm{Q} 1}=5.10 ; 95 \%$ CI 1.50-18.20 (Peeters et al, 2007)). In a US-based study, no association was observed in women diagnosed before the age of 55, but there was an inverse association in women diagnosed after the age of $55\left(\mathrm{OR}_{\mathrm{Q} 4-\mathrm{Q} 1}=0.52 ; 95 \%\right.$ CI $0.28-0.95$ (Tworoger et al, 2007)). Slightly different exclusion criteria might contribute to inconsistent results across studies (e.g., exclusion of: cases diagnosed within 1 year after blood donation (Lukanova et al, 2002), fallopian tube cancers (Tworoger et al, 2007), unilateral oophorectomy/hysterectomy (EPIC phase 1; Peeters et al, 2007). In the current study including 565 EOC cases, we observed no association between IGF-I and ovarian cancer risk regardless of the age at diagnosis.
Table 1. Selected baseline characteristics of EOC cases and matched controls at enrolment in the EPIC study

\begin{tabular}{|c|c|c|c|}
\hline & $\begin{array}{c}\text { Cases } \\
(n=565)^{a}\end{array}$ & $\begin{array}{l}\text { Controls } \\
(n=1097)^{a}\end{array}$ & $P$-value ${ }^{b}$ \\
\hline Age at blood donation ${ }^{c}$ & $57.0(33.6-80.7)$ & $56.9(33.6-79.3)$ & \\
\hline Age at diagnosis & $63.6(37.4-86.5)$ & & \\
\hline $\begin{array}{l}\text { Lag time between blood } \\
\text { donation and diagnosis }\end{array}$ & $6.7(0-16)$ & & \\
\hline \multicolumn{4}{|c|}{ Menopausal status at blood donation ${ }^{\mathbf{b}}$} \\
\hline $\begin{array}{l}\text { Pre } \\
\text { Post }\end{array}$ & $\begin{array}{l}112(20 \%) \\
453(80 \%)\end{array}$ & $\begin{array}{l}219(20 \%) \\
878(80 \%)\end{array}$ & \\
\hline Age at menopause ${ }^{d}$ & $50(32-60)$ & $50(30-59)$ & 0.03 \\
\hline Ever full-term pregnancy & & & $<0.01$ \\
\hline $\begin{array}{l}\text { No } \\
\text { Yes }\end{array}$ & $\begin{array}{r}95(17 \%) \\
448(83 \%)\end{array}$ & $\begin{array}{l}124(12 \%) \\
935(88 \%)\end{array}$ & \\
\hline OC use & & & $<0.01$ \\
\hline $\begin{array}{l}\text { Never } \\
\text { Ever }\end{array}$ & $\begin{array}{l}349(62 \%) \\
214(38 \%)\end{array}$ & $\begin{array}{l}594(54 \%) \\
498(46 \%)\end{array}$ & \\
\hline HRT use ${ }^{b}$ & & & 0.57 \\
\hline $\begin{array}{l}\text { Never } \\
\text { Ever }\end{array}$ & $\begin{array}{r}452(87 \%) \\
69(13 \%)\end{array}$ & $\begin{array}{l}867(86 \%) \\
145(14 \%)\end{array}$ & \\
\hline
\end{tabular}

\section{Histology}

\section{Serous}

Mucinous

Endometrioid

Clear Cell

NOS

Other

\section{Grade}

\section{Low grade}

High grade

\section{Stage $e^{e, g}$}

Low stage

High stage

Type I/II

Type I

Type II

IGF-I (nmoll-1) ${ }^{\mathrm{h}}$

302 (53\%)

41 (7\%)

$66(12 \%)$

28 (5\%)

99 (18\%)

29 (5\%)

Abbreviations: $\mathrm{EOC}=$ epithelial ovarian cancer; $\mathrm{EPIC}=$ European Prospective Investigation into Cancer and Nutrition; HRT = hormone replacement therapy; IGF-I = insulin-like growth factor I. Values are shown as median (range) or number (percentage).

${ }^{a}$ Cases and controls in both study phases were matched on: study recruitment centre, age at blood donation ( \pm 6 months), time of the day of blood collection ( $\pm 1 \mathrm{~h}$ ), fasting status $(<3 \mathrm{~h}, 3-6 \mathrm{~h},>6 \mathrm{~h})$ and menopausal status at blood collection (premenopausal, perimenopausal and postmenopausal), as well as menstrual cycle phase for premenopausal women ('early follicular' (days 0-7 of the cycle), 'late follicular' (days 8-11), 'peri-ovulatory' (days 12-16), 'mid-luteal' (days 20-24) and 'other luteal' (days 17-19 or days 25-40). Cases missing data on the phase of menstrual cycle were matched to controls with missing information on menstrual cycle phase.

${ }^{\mathbf{b}}$ Among postmenopausal women only.

cMatching factor.

${ }^{d}$ Differences between cases and matched controls based on conditional logistic regression.

e Percentages presented among women with data on tumour characteristics. Percentage of missing data: grade (39\%), stage (12\%) and type $1 /$ II status (45\%).

fLow-grade tumours: well differentiated tumours; high-grade tumours: moderately, poorly or undifferentiated tumours.

g Low-stage tumours: localised tumours; high-grade tumours: regional metastatic or distant metastatic tumours.

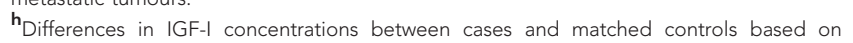
geometric mean ( $95 \%$ confidence interval); values from each study phase are standardised to a mean of 0 for analyses.

Elevated IGF-I concentrations may lead to the development of a malignant cell rather than to apoptotic cell death in the early phases of carcinogenesis (reviewed in Pollak, 2008). Insulin-like growth factor I signalling is predominantly mediated by the IGF-IR; higher IGF-IR expression is associated with development of epithelial neoplasms through anti-apoptotic and mitogenic 
Table 2. OR $(95 \% \mathrm{Cl})$ for ovarian cancer by tertiles and for doubling of IGF-I by tumour characteristics and menopausal status ${ }^{\mathrm{a}}$

\begin{tabular}{|c|c|c|c|c|c|c|c|}
\hline \multicolumn{8}{|c|}{ Tertiles $^{b}$} \\
\hline & & 1 & 2 & 3 & $\begin{array}{c}\mathrm{OR}_{\text {log2 }} \\
(95 \% \mathrm{Cl})\end{array}$ & $P_{\text {trend }}^{c}$ & $P_{\text {het }}{ }^{d}$ \\
\hline Overall & 565 sets & ref. & $0.93(0.72-1.20)$ & $0.92(0.70-1.20)$ & $0.88(0.71-1.08)$ & 0.21 & \\
\hline \multicolumn{8}{|l|}{ Histology } \\
\hline Serous & 302 sets & ref. & $1.02(0.72-1.46)$ & $1.03(0.71-1.48)$ & $0.98(0.74-1.30)$ & 0.90 & \\
\hline Mucinous & 41 sets & ref. & $1.07(0.42-2.73)$ & $0.60(0.20-1.82)$ & $0.59(0.24-1.42)$ & 0.24 & \\
\hline Endometrioid & 66 sets & ref. & $1.01(0.43-2.34)$ & $0.93(0.37-2.32)$ & $0.73(0.34-1.56)$ & 0.42 & \\
\hline Clear cell & 28 sets & ref. & $1.52(0.43-5.36)$ & $0.99(0.29-3.40)$ & $0.89(0.37-2.13)$ & 0.80 & \\
\hline NOS & 99 sets & ref. & $0.63(0.36-1.12)$ & $0.67(0.36-1.24)$ & $0.75(0.48-1.17)$ & 0.21 & \\
\hline Other & 29 sets & ref. & $0.89(0.28-2.78)$ & $1.71(0.42-6.87)$ & $1.07(0.40-2.83)$ & 0.89 & 0.12 \\
\hline \multicolumn{8}{|l|}{ Grade } \\
\hline Low grade & 35 sets & ref. & $0.24(0.06-1.08)$ & $0.56(0.15-2.00)$ & $0.87(0.34-2.24)$ & 0.78 & \\
\hline High grade & 306 sets & ref. & $0.95(0.68-1.34)$ & $1.01(0.70-1.47)$ & $0.96(0.72-1.28)$ & 0.79 & 0.89 \\
\hline \multicolumn{8}{|l|}{ Stage } \\
\hline Low stage & 76 sets & ref. & $1.23(0.57-2.67)$ & $1.39(0.65-3.01)$ & $1.02(0.56-1.85)$ & 0.95 & \\
\hline High stage & 419 sets & ref. & $0.98(0.73-1.31)$ & $0.87(0.64-1.19)$ & $0.86(0.68-1.09)$ & 0.21 & 0.52 \\
\hline \multicolumn{8}{|l|}{ Type I/II } \\
\hline Type I & 67 sets & ref. & $0.69(0.31-1.54)$ & $0.72(0.32-1.62)$ & $0.84(0.43-1.64)$ & 0.61 & \\
\hline Type II & 242 sets & ref. & $1.04(0.70-1.54)$ & $1.16(0.76-1.78)$ & $1.02(0.73-1.42)$ & 0.90 & 0.71 \\
\hline \multicolumn{8}{|c|}{ Menopausal status } \\
\hline Premenopausal & 112 sets & ref. & $0.56(0.27-1.16)$ & $0.69(0.34-1.40)$ & $0.93(0.55-1.58)$ & 0.80 & \\
\hline Postmenopausal & 452 sets & ref. & $1.01(0.77-1.32)$ & $0.93(0.70-1.26)$ & $0.87(0.69-1.08)$ & 0.21 & 0.69 \\
\hline \multicolumn{8}{|c|}{ Age at diagnosis } \\
\hline$<55$ years & 105 sets & ref. & $0.46(0.22-0.95)$ & $0.66(0.33-1.32)$ & $0.91(0.55-1.50)$ & 0.70 & \\
\hline$\geqslant 55$ years & 459 sets & ref. & $1.04(0.79-1.36)$ & $0.95(0.71-1.28)$ & $0.87(0.70-1.09)$ & 0.23 & 0.83 \\
\hline \multicolumn{8}{|c|}{ 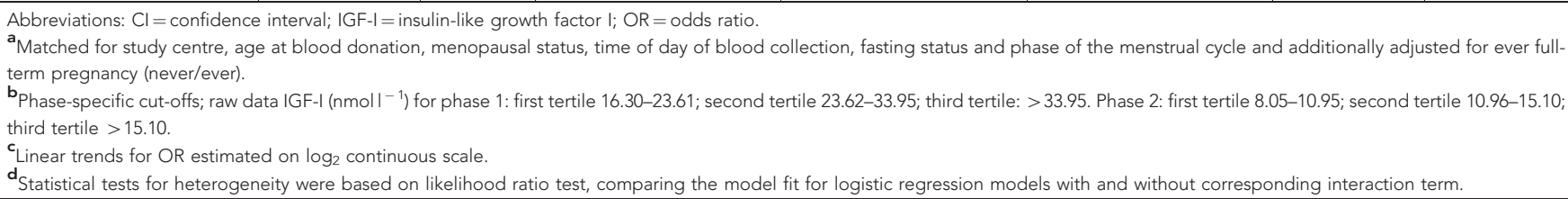 } \\
\hline
\end{tabular}

activities and its role in oncogenic transformation (reviewed in Pollak, 2008). We hypothesised that circulating IGF-I would be differentially associated with ovarian cancer subtypes given the differential expression of IGF-I in low- and high-grade serous tumours. Insulin-like growth factor I has been shown to be overexpressed in low-grade serous ovarian cancer cell lines (i.e., type I), which were more responsive to IGF-I stimulation and IGFIR inhibition compared with high-grade serous ovarian cancer cell lines (i.e., type II) (King et al, 2011). We did not observe the hypothesised associations; however, we had small sample size in some subgroups (i.e., low-grade serous tumours, $n=35$ ).

Our study has important strengths and limitations. We investigated pre-diagnostic serum IGF-I and EOC risk in a large, well-characterised nested case-control study. However, circulating IGF-I may not be reflective of IGF-I exposure in the ovary. Although the data on this association are mixed (Rabinovici et al, 1990; Thierry van Dessel et al, 1996), there is evidence to suggest that follicular fluid concentrations are well correlated with serum concentrations ( $r=0.77, P<0.001$; Dorn et al, 2003). In addition, the current analysis is based on a single biomarker in the IGF signalling axis. However, data to date suggest IGF-I and the IGF-IR are the most relevant members of the IGF family for ovarian carcinogenesis (reviewed in Beauchamp et al, 2010). In line with other epidemiologic studies, a single measurement was used to evaluate risk associations. However, relatively high intra-individual reproducibility of IGF-I measurements has been consistently shown (2-3 years; premenopausal women: $\mathrm{ICC}=0.86$ (Missmer et al, 2006), up to 5 years; pre- and postmenopausal women: $\mathrm{ICC}=0.71$ (Borofsky et al, 2002)).

The sample size for subgroup analyses by tumour characteristics (e.g., histology, grade or type I/type II) may have been too small to detect an association, with the exception of the group of serous tumours (cases $n=302$ ). For subgroup analyses by grade as well as type I/type II classification a considerable proportion of data was missing ( $>39 \%$ ), further limiting sample size in those subgroups.

Despite evidence suggesting that IGF-I could be involved in EOC development (reviewed in Bruchim and Werner, 2013; Singh et al, 2014), our study shows no association between circulating IGF-I and EOC risk. Larger, pooled prospective studies are needed to confirm our results and to address the associations in small subgroups with more statistical power and assess risk associations with expression of IGF receptors.

\section{ACKNOWLEDGEMENTS}

We thank all the EPIC participants for their invaluable contribution to the study. The German Cancer Research Center, Division of Cancer Epidemiology (Principal Investigator: Rudolf Kaaks) funded the analysis of IGF-I for this study. The European Prospective Investigation into Cancer and Nutrition is financially supported by the European Commission (DG-SANCO) and the International Agency for Research on Cancer. The national cohorts are supported by Danish Cancer Society (Denmark); Ligue Contre le Cancer, Institut Gustave Roussy, Mutuelle Générale de l'Education Nationale, Institut National de la Santé et de la Recherche Médicale (INSERM) (France); Deutsche Krebshilfe (70-2488), Deutsches Krebsforschungszentrum and Federal Ministry of Education and Research (Germany; Grant 01-EA9401); Hellenic Health Foundation (Greece) and the Stavros Niarchos Foundation; Italian Association for Research on Cancer 
(AIRC) and National Research Council (Italy); Dutch Ministry of Public Health, Welfare and Sports (VWS), Netherlands Cancer Registry (NKR), LK Research Funds, Dutch Prevention Funds, Dutch ZON (Zorg Onderzoek Nederland), World Cancer Research Fund (WCRF), Statistics Netherlands (The Netherlands); ERC2009-AdG 232997 and Nordforsk, Nordic Centre of Excellence programme on Food, Nutrition and Health. (Norway); Health Research Fund (FIS) of the Spanish Ministry of health (Exp 96/0032), Regional Governments of Andalucía, Asturias, Basque Country, Murcia (no. 6236) and Navarra, ISCIII RETIC (RD06/0020) (Spain); Swedish Cancer Society, Swedish Scientific Council and Regional Government of Skåne and Västerbotten (Sweden); Cancer Research UK, Medical Research Council (United Kingdom).

\section{CONFLICT OF INTEREST}

The authors declare no conflict of interest.

\section{DISCLAIMER}

The sponsors had no role in the study design, data collection, and analysis, interpretation of results or writing of the paper.

\section{REFERENCES}

Beauchamp MC, Yasmeen A, Knafo A, Gotlieb WH (2010) Targeting insulin and insulin-like growth factor pathways in epithelial ovarian cancer. J Oncol 2010: 257058.

Borofsky ND, Vogelman JH, Krajcik RA, Orentreich N (2002) Utility of insulin-like growth factor-1 as a biomarker in epidemiologic studies. Clin Chem 48(12): 2248-2251.

Bruchim I, Werner H (2013) Targeting IGF-1 signaling pathways in gynecologic malignancies. Expert Opin Ther Targets 17(3): 307-320.

Dorn C, Reinsberg J, Kupka M, van der Ven H, Schild RL (2003) Leptin, VEGF, IGF-1, and IGFBP-3 concentrations in serum and follicular fluid of women undergoing in vitro fertilization. Arch Gynecol Obstet 268(3): 187-193.

King ER, Zu Z, Tsang YT, Deavers MT, Malpica A, Mok SC, Gershenson DM, Wong KK (2011) The insulin-like growth factor 1 pathway is a potential therapeutic target for low-grade serous ovarian carcinoma. Gynecol Oncol 123(1): 13-18.

Kurman RJ, Shih IM (2011) Molecular pathogenesis and extraovarian origin of epithelial ovarian cancer-shifting the paradigm. Hum Pathol 42(7): 918-931.

Lukanova A, Lundin E, Toniolo P, Micheli A, Akhmedkhanov A, Rinaldi S, Muti P, Lenner P, Biessy C, Krogh V, Zeleniuch-Jacquotte A, Berrino F, Hallmans G, Riboli E, Kaaks R (2002) Circulating levels of insulin-like growth factor-I and risk of ovarian cancer. Int J Cancer 101(6): 549-554.

Maldonado G, Greenland S (1993) Simulation study of confounder-selection strategies. Am J Epidemiol 138(11): 923-936.

Missmer SA, Spiegelman D, Bertone-Johnson ER, Barbieri RL, Pollak MN, Hankinson SE (2006) Reproducibility of plasma steroid hormones, prolactin, and insulin-like growth factor levels among premenopausal women over a 2- to 3-year period. Cancer Epidemiol Biomarkers Prev 15(5): 972-978.

Ose J, Fortner RT, Schock H, Overvad K, Tjonneland A, Hansen L, Dossus L, Fournier A, Baglietto L, Romieu I, Kuhn E, Boeing H, Trichopolos D, Palli D, Masala G, Sieri S, Tumino R, Sacerdote C, Mattiello A, Ramon Quiros J, Obón-Santacanca M, Larranaga N, Chirlaque MD, Sanchez MJ, Barricarte A, Peeters PH, Bueno de Mesquita HB, Onland-Moret NC, Brändstedt J, Lundin E, Idahl A, Weiderpass E, Gram IT, Lund E, Kaw KT, Travis RC, Merritt MA, Gunter MJ, Riboli E, Kaaks R (2014) Endogenous androgens and risk of epithelial invasive ovarian cancer by tumor characteristics in the European Prospective Investigation into Cancer and Nutrition. Int J Can; e-pub ahead of print 30 May 2014; doi:10.1002/ijc.29000.

Peeters PH, Lukanova A, Allen N, Berrino F, Key T, Dossus L, Rinaldi S, van Gils CH, Bueno-de-Mesquita HB, Boeing H, Schulz M, Chang-Claude J, Linseisen J, Panico S, Sacerdote C, Palli D, Tumino R, Trichopoulou A, Trichopolos D, Bamia C, Larranaga N, Ardanaz E, Pera G, Quiros JR, Martinez-Garcia C, Navarro C, Bingham SA, Khaw KT, Clavel F, Tjonneland A, Olsen A, Overvad K, Tetsche MS, Lund E, Lundin E, Berglund G, Riboli E, Kaaks R (2007) Serum IGF-I, its major binding protein (IGFBP-3) and epithelial ovarian cancer risk: the European Prospective Investigation into Cancer and Nutrition (EPIC). Endocr Relat Cancer 14(1): 81-90.

Penson RT, Dizon DS, Birrer MJ (2013) Clear cell cancer of the ovary. Curr Opin Oncol 25(5): 553-557.

Pollak M (2008) Insulin and insulin-like growth factor signalling in neoplasia. Nat Rev Cancer 8(12): 915-928.

Rabinovici J, Dandekar P, Angle MJ, Rosenthal S, Martin MC (1990) Insulin-like growth factor I (IGF-I) levels in follicular fluid from human preovulatory follicles: correlation with serum IGF-I levels. Fertil Steril 54(3): 428-433.

Riboli E, Hunt KJ, Slimani N, Ferrari P, Norat T, Fahey M, Charrondiere UR, Hemon B, Casagrande C, Vignat J, Overvad K, Tjonneland A, Clavel-Chapelon F, Thiebaut A, Wahrendorf J, Boeing H, Trichopoulos D, Trichopoulou A, Vineis P, Palli D, Bueno-De-Mesquita HB, Peeters PH, Lund E, Engeset D, Gonzalez CA, Barricarte A, Berglund G, Hallmans G, Day NE, Key TJ, Kaaks R, Saracci R (2002) European Prospective Investigation into Cancer and Nutrition (EPIC): study populations and data collection. Public Health Nutr 5(6B): $1113-1124$

Rothman KJ, Greenland S, Lash TL (2008) Modern Epidemiology. 3rd edn. Wolters Kluwer Health/Lippincott, Williams \& Wilkins: Philadelphia.

Singh P, Alex JM, Bast F (2014) Insulin receptor (IR) and insulin-like growth factor receptor 1 (IGF-1R) signaling systems: novel treatment strategies for cancer. Med Oncol 31(1): 805.

Thierry van Dessel HJ, Chandrasekher Y, Yap OW, Lee PD, Hintz RL, Faessen GH, Braat DD, Fauser BC, Giudice LC (1996) Serum and follicular fluid levels of insulin-like growth factor I (IGF-I), IGF-II, and IGF-binding protein-1 and -3 during the normal menstrual cycle. J Clin Endocrinol Metab 81(3): 1224-1231.

Tworoger SS, Lee IM, Buring JE, Pollak MN, Hankinson SE (2007) Insulin-like growth factors and ovarian cancer risk: a nested casecontrol study in three cohorts. Cancer Epidemiol Biomarkers Prev 16(8): 1691-1695.

This work is published under the standard license to publish agreement. After 12 months the work will become freely available and the license terms will switch to a Creative Commons AttributionNonCommercial-Share Alike 3.0 Unported License. 